\title{
Prospective Single-Arm, Multi-Center Trial of a Patient-Specific Interpositional Knee Implant: Early Clinical Results
}

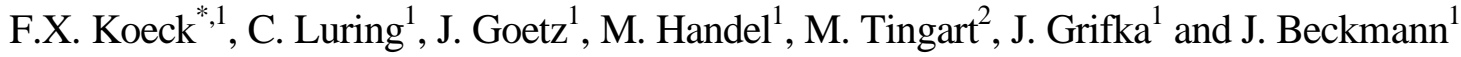 \\ ${ }^{I}$ Department of Orthopaedic Surgery, University of Regensburg, Germany \\ ${ }^{2}$ Department for Orthopaedics and Trauma Surgery, University of Aachen, Germany
}

\begin{abstract}
Background: The treatment of unicompartmental arthritis in younger patients is challenging. The aim of this study is to report final safety and efficacy analysis results for the iForma patient-specific interpositional device, which is designed for the treatment of isolated medial or lateral compartment arthritis of the knee.

Methods: From June 2005 to June 200878 subjects ( 42 men, 36 women) received an iForma implant. The mean age was 53 years, the mean Body Mass Index 29.0. We surveyed the WOMAC scores, the visual analog pain scale and the Knee Society Scores.

Results: The mean follow up was 16.4 months. The mean WOMAC knee scores increased from 48.3 before surgery to 71.3 after 24 months. A reduction in pain was achieved for all five pain measures using a standard visual analog scale (VAS). Knee Society Knee Score improved from 39.2 before to 61.924 month after surgery. The Knee Society Function Scores improved form preoperative 64.5 to 82.52 years postoperative. The preoperative range of motion could be restored. The overall revision rate was $24 \% .15$ implants were removed early, 4 knees were revised without implant removal.

Conclusion: Within narrow indication of patients with unicompartmental disease, the iForma device can provide improvement in knee function and reduction in pain, however, with a significant higher risk of early revision compared to traditional arthroplasty. Respecting this limitation it may be an alternative option for arthritic patients with unicompartmental disease who have contraindications to High Tibial Osteotomy or are too young for knee replacement; the iForma device further has the distinct advantage of time and cost saving compared to those procedures.
\end{abstract}

Keywords: Unicompartmental knee arthritis, Hemiarthroplasty, Interpositional implant, patient-specific.

\section{INTRODUCTION}

Orthopedic surgeons see increasing numbers of younger (i.e. <60 years), more active patients with unicompartmental osteoarthritis of the knee. These patients have passed the age for biological cartilage repair, but are considered too young for traditional, more invasive joint replacement. A typical patient profile includes those who have undergone one or more arthroscopies for mechanical symptoms of the knee and who suffer from recurrent pain related to cartilage loss and early osteoarthritis. The treatment of middle-aged, active patients, however, is challenging [1]. For patients who continue to have disabling pain and interference with daily activities in spite of conservative treatment, a less invasive surgical intervention may be indicated. Micro-fracture is generally not recommended in patients over 40 years of age $[2,3]$. HTO can correct malalignment, but it involves a highly invasive surgical procedure that may pose problems during later knee replacement $[4,5]$. Arthroplasty provides good pain relief for most patients, either total-knee arthroplasty (TKA) or unicompartmental knee arthroplasty (UKA). Both, however, require significant bone resection.

*Address correspondence to this author at the Department of Orthopedic Surgery, University of Regensburg, Kaiser-Karl-V-Allee 3, D-93077 Bad Abbach, Germany; Tel: ++49-9405-180; Fax: ++49-9405-18-2925;

E-mail: franz.koeck@klinik.uni-regensburg.de
For patients with osteoarthritis limited to a single femoral-tibial compartment, the concept of a metallichemiarthroplasty has a long history. Having been introduced in the 1950's by MacIntosh and McKeever [6, 7], the approach gained only limited use within the orthopedic community due to its invasive nature and the advent of total knee replacement. Nonetheless, the reports of early experience with this technique were generally encouraging $[6,7]$. More recent clinical reports by Scott and colleagues [8] and Emerson and Potter [9] also reported good results. The most recent report of long-term results, published by Springer, Scott and colleagues in 2006 [10] continued to show excellent long-term results with tibial hemiarthroplasty using the McKeever device. However, contrary outcomes have been reported with the Unispacer ${ }^{\mathrm{TM}}$ knee system (Zimmer, Warsaw, IN, USA) with, in some studies, high failure rates at 6 months and after, ranging between 16 and 44 percent $\mathrm{t}$ [11-14].

In contrast to the Unispacer, the iForma is a patientspecific interpositional device. The undersurface of the implant replicates the tibial articular surface resulting in functional fixation similar to a McKeever device. The top surface conforms to the shape of the femoral condyle (Fig. 1). The implant is developed from a standard MRI scan using a novel image-to-implant technology that converts the topography of the patient's articular cartilage and 
subchondral bone to a patient-specific implant. Goal of the present study was to find out, whether an individualized interpositional device is a valuable option for treatment of unicompartmental knee arthritis. We evaluated the safety and clinical efficacy of the iForma implant in a single-arm multicenter study in the United States and Europe in 78 patients. Western Ontario MacMaster (WOMAC) scores [15, 16], Visual Analog Scale (VAS) of pain [17, 18] and the Knee Society Scores [19] were measured at different time intervals over a 2-year period.

\section{MATERIALS AND METHODS}

\section{Subjects}

The prospective single-arm, multi-center study was reviewed and approved by a central Institutional Review Board in the United States and local ethics commissions in Europe (IRB No. 04/75, Regensburg University) in 2004. Before being enrolled in the study, all subjects gave written informed consent to participate. During the period from June 2005 through June 2008, 78 subjects were enrolled at four sites in the USA and five sites in Germany. At each site one single surgeon, who had successfully completed bioskills training and had implanted at least three iForma interpositional devices, determined the indication and performed the procedures. Indications, contraindications, as well as inclusion and exclusion criteria used for trial enrollment, are shown in Table $\mathbf{1}$.

Data was collected at each site according to the schedule of assessment in Table 2, and documented on paper Case Report Forms (CRFs). Various assessment tools were employed in this study to assess the pain and function after surgery.

The WOMAC knee index [15] is a patient-administered questionnaire which assesses three dimensions of pain, disability and joint stiffness. We used the reduced WOMAC function scale which transforms the raw WOMAC function score to a 0 to 100 scale, worst to best. A score of 0 indicates extreme restriction in all activities, and a score of 100 indicates no restriction for any item [16].

The Knee Society rating system (KSS) [19] is a commonly used tool which combines a knee score that rates the knee joint only and functional score that rates the subject's ability to walk and climb stairs. The KSS Knee Score and Function Score range from 0 to 100 with 0 being worst and 100 being best.

Subjects were examined and questioned regarding the occurrence of adverse events at each follow-up visit.

In addition to routine radiographic images, pre-operative MRI scans were acquired using a protocol consisting of coronal and sagittal cartilage-sensitive pulse sequences with $3.5 \mathrm{~mm}$ slice-thickness. MR images were evaluated by the surgeon and an independent radiologist delegated by the manufacturer. The MRI scans and the radiologist analysis were also used to assess each subject for indications and contraindications as specified in Table 1. An individualized medial (Fig. 1) or lateral interpositional implant was constructed based on the patient's MRI by ConforMIS, Inc., (Burlington, MA 01803) using proprietary image-to-implant software and then manufactured using a CAD/CAM process.

Table 1. Indications/Inclusion Criteria and Contraindications/Exclusion Criteria

\begin{tabular}{|c|c|}
\hline Indications/Inclusion Criteria & Contraindications/Exclusion Criteria \\
\hline $\begin{array}{l}\text { - Isolated medial or lateral osteoarthritis of the knee } \\
\text { - Cartilage damage in opposite compartment grades 0, I or II, asymptomatic } \\
\text { - Cartilage damage in the patellar-femoral compartment grades } 0, \text { I or II, } \\
\text { asymptomatic } \\
\text { - Age between } 35 \text { and } 65 \text { years } \\
\text { - Intact subchondral bone plate }\end{array}$ & $\begin{array}{l}\text { - Severe patellofemoral or tibiofemoral osteoarthritis in the } \\
\text { opposite compartment } \geq \text { grade III } \\
\text { - Severe articular instability caused by loss of osteochondral } \\
\text { structures, e.g. femoral flattening } \\
\text { - Ligament instability or loss of collateral or cruciate ligament } \\
\text { integrityFlexion contracture }>15 \mathrm{deg} \text {. } \\
\text { - Generalized bone disease, including osteoporosis, Paget's disease } \\
\text { or other metabolically induced bone disease } \\
\text { - Presence of active infection }\end{array}$ \\
\hline
\end{tabular}

Table 2. Visit Schedule of Assessments

\begin{tabular}{|c|c|c|c|c|c|c|c|}
\hline Visit & Pain (VAS) & Medication & Knee Society Health Survey & Knee Evaluation & X-Ray & Operative Data & WOMAC Survey \\
\hline \hline Pre-op & $X$ & $X$ & $X$ & $X$ & $X$ & $X$ \\
\hline Intra-op & & & & & $X$ & $X$ \\
\hline Discharge & $X$ & $X$ & $X$ & & & \\
\hline 6 Weeks & $X$ & $X$ & $X$ & $X$ & & & \\
\hline 3 months & $X$ & $X$ & $X$ & $X$ & $X$ & & \\
\hline 6 months & $X$ & $X$ & $X$ & $X$ & $X$ & & \\
\hline 12 months & $X$ & $X$ & $X$ & $X$ & $X$ & \\
\hline 24 months & $X$ & $X$ & $X$ & $X$ & $X$ \\
\hline
\end{tabular}




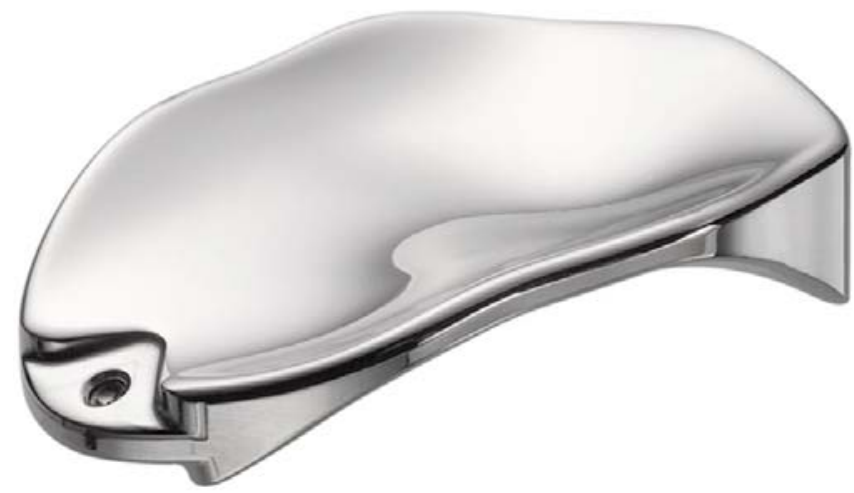

Fig. (1). Patient specific interpositional device.

\section{Surgical Technique and Post-Operative Care}

In all cases, as the first step of a one-stage-prodedure, an arthroscopic resection of the posterior horn of the meniscus, leaving a $2 \mathrm{~mm}$ narrow seam, was performed. The compartment was opened with a medial or lateral parapatellar incision using a minimally invasive technique. The anterior horn of the meniscus was mobilized and completely removed up to the dorsal resection boundary with the menisectomy ensuring the preservation of capsular as well as further soft tissue integrity. With a Luer rasp and a small file, any existing peripheral femoral and tibial osteophytes were removed. Following that, the device was inserted using a grasper (Fig. 2).

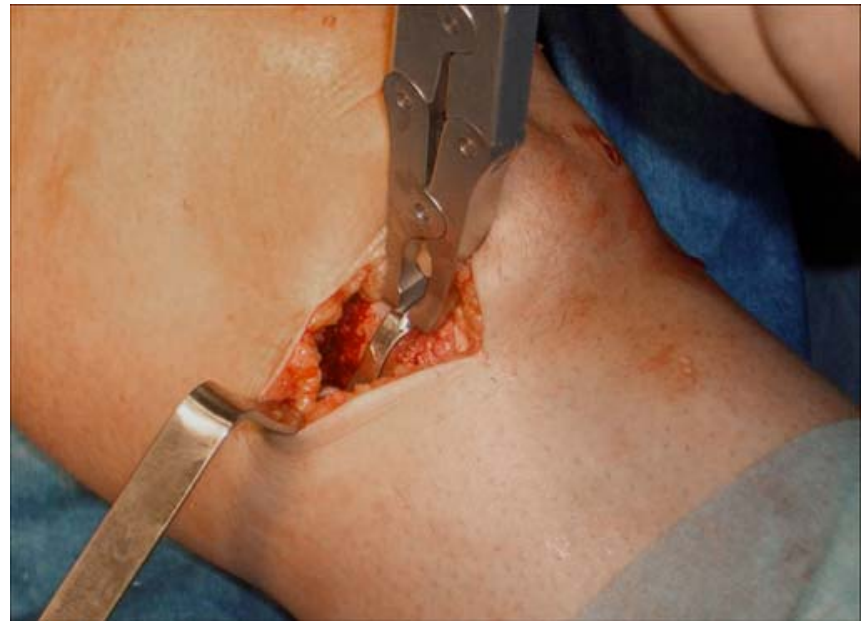

Fig. (2). Interpositional device being inserted using the grasper.

Medial implants were inserted starting from a $45^{\circ}$ flexed position with valgus stress applied to the knee joint from antero-medial to dorso-lateral using a slight tilting motion. Lateral implants were inserted starting with a $45^{\circ}$ tilt of the implant strictly from lateral to medial with simultaneous varus stress applied. Implant stability and range of motion were verified visually, by palpation, and by dynamic fluoroscopy (Fig. 3).

Closure of the wound was performed in several layers using standard technique. Drains were placed at the discretion of the individual surgeon.

Subjects were discharged with instructions for pain management during the first 2 days in the US and 5 days in Germany, including the use of ice, NSAIDs or prescribed pain medication. Limited activity was permitted upon discharge, with weight bearing permitted as tolerated. Passive motion was not used postoperatively. Physical therapy or directed activity was recommended according to the individual surgeon's preference. Usually no postoperative bracing was used.

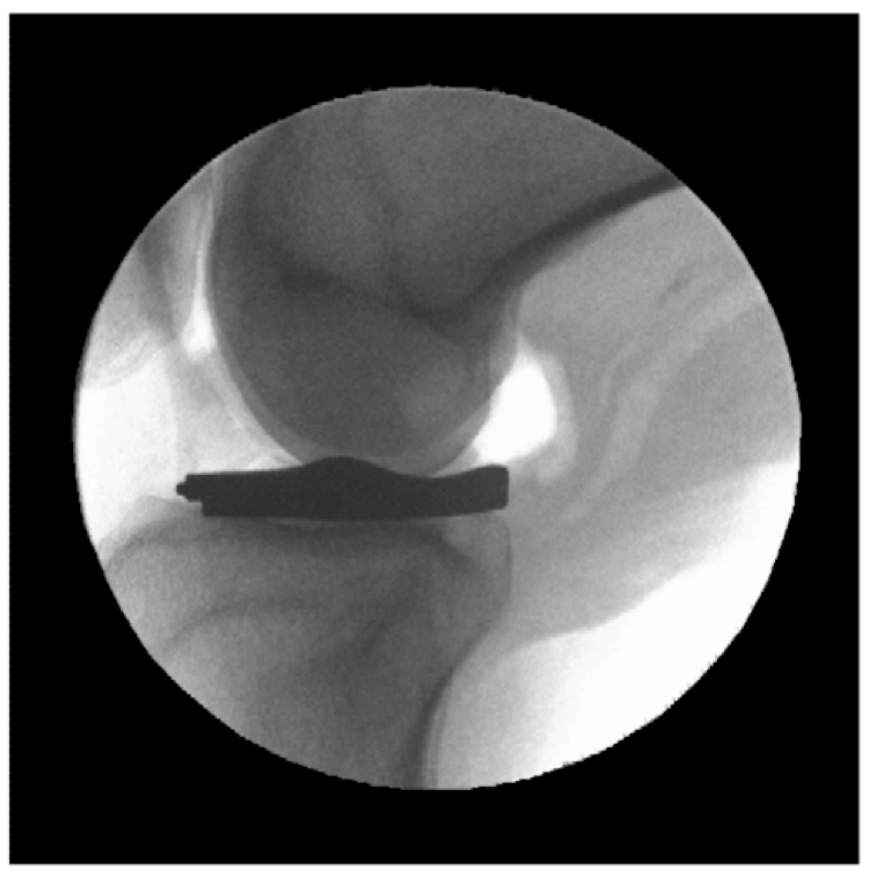

Fig. (3). Fluoroscopy of interpositional device placement and range of motion.

\section{Statistical Methods}

All data related to the study was summarized using standard descriptive statistics. The analysis of variance (ANOVA) methods for repeated measurements and Tukey's multiple comparison tests as a post-hoc analysis were used for comparing means of WOMAC and VAS scores for all patient visits. Estimates of mean differences and their 95\% confidence intervals were presented. The study hypotheses were tested on the level of alpha $=5 \%$ throughout the analysis. Bonferroni adjustments were made for multiple comparisons. All statistical analyses comparing mean scores for all visits were performed using SAS statistical software, version 9.1 (SAS Institute, Cary, NC, U.S.A.) and graphs were plotted using R-software (The R-Foundation for Statistical Computing).

\section{RESULTS}

At the time the study was voluntarily terminated (September 2009), 78, 73, 72, 58, 44, and 26 patients had completed their visits at the preoperative, 1 month, 3 month, 6 month, 12 month and 24 month follow-up intervals, respectively (Table $\mathbf{3}$ ).

The mean follow up was 16.4 months. Of the 78 implants, $72(92 \%)$ were medial, and $6(8 \%)$ lateral. The mean age at the time of surgery was 53 years (range 37-71 years; 1 subject was entered outside the specified range as a protocol exception). The mean BMI was 29.0 (14.7 to 43.3, SD 5.47); $52 \%$ of patients were male, $48 \%$ of the patients were female. All patients had arthroscopy before the procedure. Using the Outerbridge classification [20] cartilage 
Table 3. Example of MRI Protocol Used for Generating Patient-Specific iForma Implant Design Using 1.5 Tesla Magnets

\begin{tabular}{|c|c|c|c|c|c|c|c|c|c|c|}
\hline Images Acquired & & Plane & Pulse Sequence & TR & TE & Slice Thickness & Spacing & Matrix & FOV & Voxel Size \\
\hline Required & 1 & Sagittal & Proton Density FS & $\sim 4500$ & $\sim 15 \mathrm{msec}$ & $3 \mathrm{~mm}$ & 0.5 & $512 \times 256$ & $14-16 \mathrm{~cm}$ & $\sim .27 \mathrm{~mm}$ \\
\hline Alternative & 3 & Sagittal & 3D- SPGR FS & $\sim 60$ & $\sim 5 \mathrm{msec}$ & $3 \mathrm{~mm}$ & 0.5 & $512 \times 192$ & $14-16 \mathrm{~cm}$ & $\sim .27 \mathrm{~mm}$ \\
\hline Alternative & 3 & Coronal & 3D-SPGR FS & $\sim 60$ & $\sim 5 \mathrm{msec}$ & $3 \mathrm{~mm}$ & 0.5 & $512 \times 192$ & $14-16 \mathrm{~cm}$ & $\sim .27 \mathrm{~mm}$ \\
\hline
\end{tabular}

was rated grade III in $22 \%$, and grade IV in $74 \%$ of the affected compartments.

The mean WOMAC-Score increased from 48.3 before surgery to 68.9 at 6 months. Improvement in the reduced WOMAC score at 6 months remained consistent and statistically significant through 24 months (Table 4).

The Knee Society Knee Scores increased steadily after surgery through the 24 month follow-up visit, with the largest increase of $22 \%$ occurring between the pre-operative and the 1 month/6 week follow-up visit $(\mathrm{p}<0.05)$ (Table 4).

The Knee Society Function Scores (subject's ability to walk and climb stairs) demonstrated a decrease immediately after the implant surgery at the 1 month/6 week follow-up visit due to the recovery period from the surgery. However, the Knee Society Function scores steadily increased after the 1 month/6 week follow-up visit through 24 month follow-up. By 24 months, the mean Knee Society Function Score had increased by $28 \%$ when compared to the pre-operative mean Knee Society Function Score $(\mathrm{p}<0.05)$. The mean Knee Society Function Score demonstrated an improvement in the subject's ability to participate in various activities (Table 4) as well as an improvement in subject's ability to walk without supportive devices (Table 4). At baseline, $62 \%$ of the subjects reported only being capable of minimal walking or light housework/office work and $76 \%$ of subjects were able to walk only up to one hour without supportive devices. By their 24 month follow-up visit, $92 \%$ of the subjects reported being able to do at heavy housework/light exercise or moderate to vigorous sports and $69 \%$ of the subjects were able to walk for over one hour without supportive devices (Table 4).

Statistically significant reduction in pain as measured by the WOMAC Pain Scores was achieved for all five pain measures following the procedure $(\mathrm{p}<0.05)$. Pain decreased steadily, immediately following the surgery as demonstrated by VAS scores at discharge, and continued to decrease through the 24 month follow-up visit (Fig. 4).

The only exception was for the activity of "standing" the VAS score demonstrated a slight increase from 2.1 at the 12 month follow-up visit to 2.3 at the 24 month follow-up visit. The greatest improvements in pain were seen between the pre-op and discharge visits which may be related directly to the surgical implant of the interpositional device, and between the 3 month and 6 month visits which may be related to the time needed by the subjects to fully recover from the surgery. Remarkably, VAS pain scores decreased significantly $(\mathrm{p}<0.05)$ from the preoperative level at the time of discharge for the three active state evaluations (walking, standing upright and walking down stairs). Pain while sitting or lying down was significantly improved by 1 month and pain in bed at night achieved significant improvement by 3 months. Pain improvement was maintained over the course of the study with no statistically significant difference in VAS pain scores between the 3, 6, 12 and 24 month intervals.

While subjects demonstrated an expected decrease in range of motion immediately after surgery and during their recovery period, on average the subjects were able to return to their pre-op range of motion by their 12 month follow-up visit. The preoperative slight mean flexion contracture of $0.1^{\circ}$ (SD 3.67) increased to a maximum of $2.0^{\circ}$ (SD 17.45) at 6 months postoperative, came back to $0.2^{\circ}$ (SD 2.42) at 12 months and stayed at the same level $\left(0.2^{\circ}\right.$, SD 3.53) until 24 months. The maximum knee flexion of preoperative $129.5^{\circ}$ (SD 20.43) decreased to $84.6^{\circ}$ at the time of discharge and steadily increased to $120.9^{\circ}$ (SD 22.66) at 6 months, $128.7^{\circ}$ (SD 13.57) at 12 months and $130.9^{\circ}$ (SD 16.56) at 24 months postoperative.

Of the 78 patients, there were 15 (19\%) explants and 4 (5\%) surgical interventions without implant removal. These surgical interventions included re-arthroscopies for pain,

Table 4. Mean WOMAC Scores, Knee Society Knee/Function Scores and Subjects' Ability to Walk

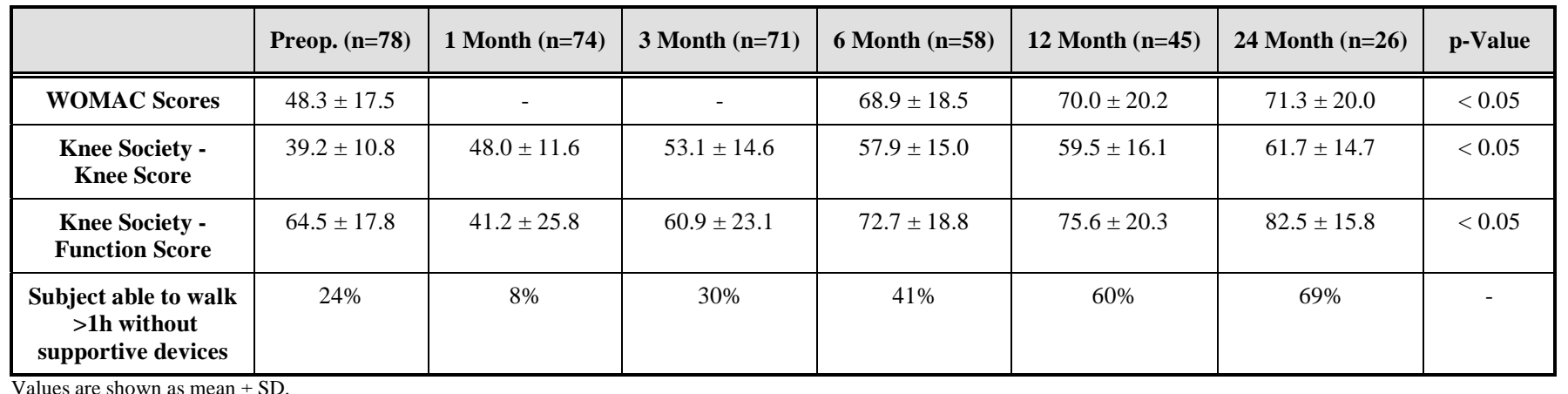

\footnotetext{
Values are shown as mean \pm SD
} 


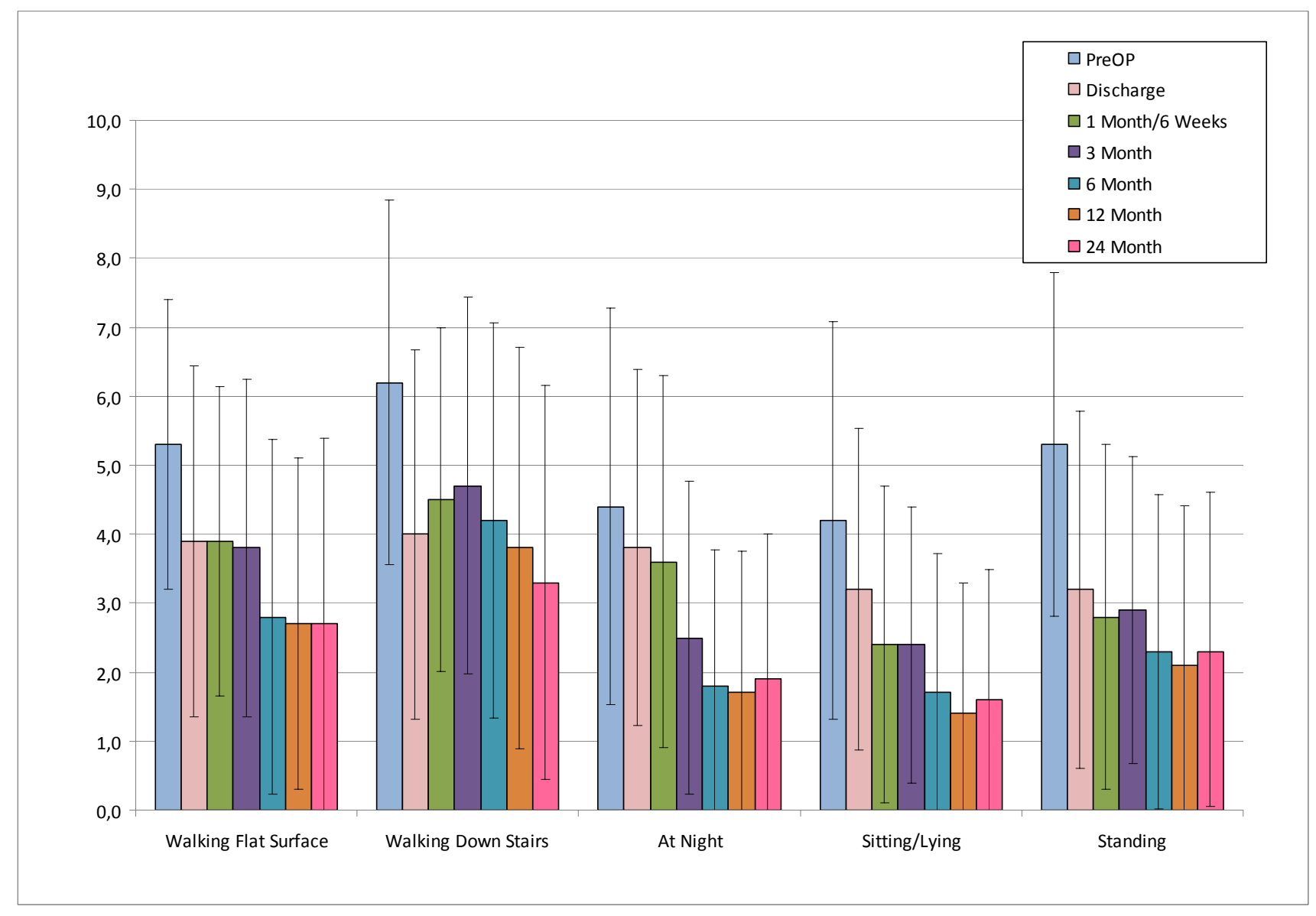

Fig. (4). Mean WOMAC Pain Scores for various activities.

instability or dislocation. The total revision rate of $24 \%$ is determined by combining the explants and surgical interventions. See Table 5 for a summary of the events leading to explants and surgical interventions.

Table 5. Number of Explants and Surgical Interventions

\begin{tabular}{|c|c|c|c|}
\hline Adverse Event & $\begin{array}{c}\text { No. of } \\
\text { Occurences }\end{array}$ & Explant & $\begin{array}{c}\text { Surgical } \\
\text { Intervention }\end{array}$ \\
\hline \hline Persistent pain & 9 & 7 & 2 \\
\hline Subluxation & 2 & 2 & - \\
\hline Dislocation & 3 & 2 & 1 \\
\hline Instability & 1 & 1 & 1 \\
\hline Limp & 1 & 1 & - \\
\hline Implant impingement & 1 & 1 & - \\
\hline Infection & 1 & 1 & - \\
\hline Allergy concern & 1 & 1 & - \\
\hline Total & $\mathbf{1 9}$ & $\mathbf{1 5}$ & $\mathbf{4}$ \\
\hline
\end{tabular}

\section{DISCUSSION}

Clinical results with unicompartmental arthroplasty have generally been reported to be good. Cartier and Cheaib [21] reported two decades ago on a series of 159 Mod $U$ unicompartmental arthroplasties with a mean follow-up of 54 months with $91 \%$ excellent clinical results and $6 \%$ failures. In a more recent study, Furnes and colleagues evaluated failure and survival rates for unicompartmental and total knee implants based on a decade of data in the Norwegian Arthroplasty records [22]. The authors found that unicompartmental devices had an approximate incidence of failure of $3 \%$ in the first year and $6 \%$ after two years.

The experience with the Unispacer device, however, has been quite different. Clinically high dislocation rates have been reported in the literature at time intervals as early as 6 months after surgery $[11,12]$. The unispacer represented a new concept of a free floating interpositional metallic implant that actively translates in anteroposterior direction and rotates with knee flexion and extension. The reported failure rates have resulted in limited, if any, use of the device today [11].

The principle of functional implant stabilization of the iForma device which is matching the individual joint surface however is more similar to the principle of fixed tibial hemiarthroplasty using the concepts first described by Macintosh and McKeever [6, 7]. Scott [8] and Springer [10] published good long-term results with the McKeever device, within a narrow, well-defined indication of patients with unicompartmental disease and intact ligaments. 
The incidence of implant revision with the iForma in this study was $24 \%$, primarily due to persistent pain and implant subluxation/dislocation. This failure rate is above revision rates for traditional unicompartmental arthroplasty [21, 22]. But unlike traditional arthroplasty failure modes with bone loss, osteolysis and implant loosening, failure of the iForma implant is different. The device can simply be removed. Since no bone or cartilage has been resected, the patient can still go on to UKA or primary TKA.

As shown in Table 3, there was a significant difference between complication rates of experienced surgeons who performed more than 10 iForma -implantations and inexperienced surgeons who did fewer than 10 implantations. Whereas the experienced surgeons had an overall revision rate (Termination early due to adverse event) of $16 \%$, the inexperienced surgeons had a revision rate almost three times higher (45\%). Similar effects were shown by Hallock et al. for the Unispacer [13, 14], who found a marked decrease in complication rates between a first group of 79 and a second group of 78 patients, and also in follow-up studies on UKA [23, 24].

The rates of revision for pain and revision for subluxation/dislocation observed in the present study are comparable to the reported overall one-year revision rate of $21 \%$ for the Unispacer in an early report [13] but lower than the number of $32 \%$ reported in two-year follow-up [14]. In the second published report the incidence of revision for the Unispacer for dislocations was $16 \%$ [14], compared with $4.8 \%$ for all cases (including improper surgical technique) in the current study, and $16 \%$ for persistent pain, compared with $2.4 \%$ for the iForma implant in the current study. In addition, the implant design of the Unispacer was intended only for use in the medial compartment [13]. In contrast, the iForma makes use of functional fixation and therefore can be used for either the medial or lateral compartment.

Patients reported a rapid partial recovery, followed by 48 weeks or more of continued recovery with full benefit typically seen at 3 months. Joint effusions were observed between 4 and 8 weeks after surgery, and typically resolved 3 months postoperatively. The improvement in WOMAC scores compares favorably with the improvement seen in subjects who receive a fixed-bearing or mobile-bearing unicompartmental arthroplasty [25] or TKA [26]. Subjects with the iForma tend to experience a "wear-in" period lasting 3 months which is followed by a return to full pre-operative range of motion within 6 months to 1 year and a continued trend of improved pain relief out to 2 years.

Limitations of the study are the lack of a control group, the just short- to medium-term follow-up and the involvement of many centers with just few implantations each which. The latter might have influenced the overall relatively high rate of failures, possibly depicting a learning curve and further highlighting the careful patient selection. However, although we can only report preliminary results with 2 years of follow-up, we consider the iForma to offer a potentially valuable option to younger subjects who may not be candidates for a HTO or more invasive arthroplasty options, such as a UKA or TKA. The device should only be used in patients with unicompartmental disease, who have intact ligaments, an intact subchondral bone plate on both the femur and tibia and who suffer from an intra-articular knee deformity, which reliably can be corrected with this implant as already shown [27].

During the period of the study, the cost of the iForma implant in the US and in Germany was similar to the cost of a standard unicompartmental implant, although variations in price of competing systems at each institution varied. Taking into account that the time of surgery for the iForma was significantly lower and the cost for instrumentation and sterilization of the instruments was minimal compared to a conventional UKA or TKA, we consider it to be a comparable to the cost of other implant options.

\section{CONCLUSION}

The preliminary two-year results of the iForma implant in this single-arm, multi-center trial are demonstrating improvement in both knee function and pain relief in a population that was significantly younger than typical candidates for UKA or TKA. However, the relatively high failure rate highlights careful patient selection.

Within a narrow, well-defined indication, in our opinion the iForma offers a potentially valuable option to patients who are border candidates for more invasive arthroplasty options or a HTO. A key benefit of the iForma device over traditional unicompartmental arthroplasty systems is the preservation of cartilage and bone. When the iForma device fails, it can be simply extracted.

\section{ACKNOWLEDGEMENT}

The authors are grateful to Dr. Sudesh Srivastav, Department of Biostatistics, Tulane University, New Orleans, for his help in performing the statistical analyses.

\section{CONFLICT-OF-INTEREST-POLICY}

The authors did receive outside funding in support of their research from ConforMIS Inc. (Burlington USA).

\section{REFERENCES}

[1] Iorio P, Healy WL. Unicompartmental arthritis of the knee. J Bone Joint Surg Am 2003; 85-A: 1351-64.

[2] Rodrigo JJ, Steadman JR, Silliman JF, Fulstone HA. Improvement of full-thickness chondral defect healing in the human knee after debridement and microfracture using continuous passive motion. Am J Knee Surg 1994; 7: 109-16.

[3] Bievina FT, Steadman JR, Rodrigo JJ, Sillman J. Treatment of articular cartilage defects in athletes: an analysis of functional outcome and lesion appearance. Orthopedics 1998; 21: 761-8.

[4] Coventry MB, Ilstrup DM, Wallrichs SL. Proximal tibial osteotomy: A critical long-term study of eighty-seven cases. J Bone Joint Surg Am 1993; 75-A: 196-201.

[5] Insall J, Joseph DM, Msika C. High tibial osteotomy for varus gonarthrosis: A long-term follow-up study. J Bone Joint Surg Am 1984; 66-A: 1040-8.

[6] Macintosh DL. Hemi-arthroplasty of the knee using a space occupying prosthesis for painful varus and valgus deformities. Proceedings of the joint meeting of orthopaedic associations of the English speaking world. J Bone Joint Surg Am 1958; 40-A: 1431.

[7] McKeever DC. Tibial plateau prosthesis. Clin Orthop 1972; 18: 8695.

[8] Scott RD, Joyce MS, Ewald FC, Thomas WH. McKeever metallic hemiarthroplasty of the knee in unicompartmental degenerative arthritis. J Bone Joint Surg Am 1985; 67-A: 203-7.

[9] Emerson R, Potter T. The use of the McKeever metallic hemiarthroplastsy for unicompartmental arthritis. J Bone Joint Surg Am 1985; 67-A: 2008-12.

[10] Springer BD, Scott RD, Sah AP, Carrington R. McKeever hemiarthroplasty of the knee in subjects less than sixty years old. J Bone Joint Surg Am 2006; 88-A: 366-71. 
[11] Bailie AG, Lewis PL, Brumby SA, Roy S, Paterson RS, Campbell DG. The Unispacer knee implant: Early clinical results. J Bone Joint Surg Br 2008; 90-B: 446-50.

[12] Sisto DJ, Mitchell IL. UniSpacer arthroplasty of the knee. J Bone Joint Surg Am 2005; 87-A: 1706-11.

[13] Hallock RH, Fell BM. Unicompartmental tibial hemiarthroplasty: early results of the UniSpacer knee. Clin Orthop Relat Res 2003: 416: $154-63$

[14] Hallock RH. The UniSpacer: A Treatment alternative for the middle-aged patient. Orthop Clin North Am 2005; 36: 505-12

[15] Bellamy N, Buchanan WW, Goldsmith CH, Campbell J, Stitt LW. Validation study of WOMAC: a health status instrument for measuring clinically important subjects relevant outcomes to antirheumatic drug therapy in subject with osteoarthritis of the hip or knee. J Rheumatol 1988; 15: 1833-40.

[16] Whitehouse SL, Lingard EA, Katz JN, Learmonth ID. Development and testing of a reduced WOMAC function scale. J. Bone Joint Surg Br 2003, 85: 706-11

[17] Katz J, Melzack R. Measurement of pain. Surg Clin North Am 1999; 79(2): 231-52.

[18] Scott J, Huskisson EC. Graphic representation of pain. Pain 1976; 2: $175-84$

[19] Insall JN, Dorr LD, Scott RD, Scott WN. Rationale of the Knee Society clinical rating system. Clin Orthop Relat Res 1989; 248: $13-4$.
[20] Outerbridge RE. The etiology of chondromalacia patellae. J Bone Joint Surg Am 1961; 43-A: 752-57.

[21] Cartier P, Cheaib S. Unicondylar knee arthroplasty: 2-10 years of follow-up evaluation. J Arthoplasty 1987; 2: 157-62.

[22] Furnes O, Espehaug B, Lie SA, Vollset SE, Engesæter, SB, Havelin LI. Failure mechanisms after unicompartmental and tricompartmental primary knee replacement with cement. J Bone Joint Surg Am 2007; 89-A: 519-25.

[23] Hamilton WG, Ammeen D, Engh CA, Engh GA. Learning curve with minimally invasive unicompartmental knee rthroplasty. J Arthroplasty 2010; 25 (5): 735-40.

[24] Harnett NI; Tregonning R; Rothwell A; Hobbs T. The Oxford Phase 3 unicompartmental knee arthroplasty - an audit of revisions. The New Zealand Experience. J Bone Joint Surg Br 2010; 92-B, Supp I: 224.

[25] Li MG, Yao F, Joss B, Ioppolo J, Nivbrant B, Wood D. Mobile vs fixed bearing unicondylar knee arthroplasty: A randomized study on short term clinical outcomes and knee kinematics. Knee 2006; 5: 365-70.

[26] Kane RL, Saleh KJ, Wilt TJ, Bershadsky B. The functional outcomes of total knee arthroplasty. J Bone Joint Surg Am 2005; 87-A(8): 1719-24.

[27] Koeck FX, Perlick L, Luring C, et al. Leg axis correction with ConforMIS iForma (interpositional device) in unicompartmental arthritis of the knee. Int Orthop 2009; 33(4): 955-60.

(C) Koeck et al.; Licensee Bentham Open.

This is an open access article licensed under the terms of the Creative Commons Attribution Non-Commercial License (http://creativecommons.org/licenses/by$\mathrm{nc} / 3.0 /$ ) which permits unrestricted, non-commercial use, distribution and reproduction in any medium, provided the work is properly cited. 